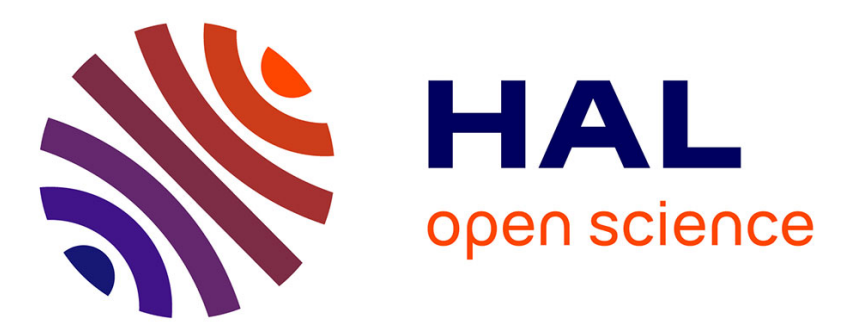

\title{
Multidimensional systems: BIBO stability test based on functional Schur coefficients
}

\author{
Ioana Serban, Mohamed Najim
}

\section{To cite this version:}

Ioana Serban, Mohamed Najim. Multidimensional systems: BIBO stability test based on functional Schur coefficients. IEEE Signal Processing Magazine, 2007, 55 (11), pp.5277 - 5285. 10.1109/TSP.2007.896070 . hal-00181756

\section{HAL Id: hal-00181756 https://hal.science/hal-00181756}

Submitted on 24 Oct 2007

HAL is a multi-disciplinary open access archive for the deposit and dissemination of scientific research documents, whether they are published or not. The documents may come from teaching and research institutions in France or abroad, or from public or private research centers.
L'archive ouverte pluridisciplinaire HAL, est destinée au dépôt et à la diffusion de documents scientifiques de niveau recherche, publiés ou non, émanant des établissements d'enseignement et de recherche français ou étrangers, des laboratoires publics ou privés. 


\title{
Multidimensional Systems: BIBO Stability Test Based on Functional Schur Coefficients
}

\author{
Ioana Serban and Mohamed Najim, Fellow, IEEE
}

\begin{abstract}
This paper presents a multidimensional extension of the Schur-Cohn algorithm for testing BIBO stability in $N$ variables. This new method only needs a unique condition to be checked, as an alternative to the set of $N$ conditions of the well-known Huang-Jury-Anderson stability test. The proposed algorithm is based on a generalization of the Schur coefficients recently obtained by the authors. In the two-variable case a simplification to the Sturm algorithm is presented, which can also be used in the case of a polynomial with complex coefficients. This approach is illustrated on several numerical examples.
\end{abstract}

Index Terms-BIBO stability, functional Schur coefficients, multidimensional Schur-Cohn test, positive trigonometric polynomials.

\section{INTRODUCTION}

$\mathbf{T}$ HE problem of testing BIBO stability of multivariable systems is a quite difficult issue, unlike the one-variable case where the solution is given by the well-known Schur-Cohn algorithm [15]. In order to recall the definition of BIBO stability, let us denote by $A$ and $P$ two coprime polynomials in $N$ variables with complex coefficients, $\mathbb{D}$ the open unit disk in the complex plane, $\mathbb{T}$ the unit circle, and $\overline{\mathbb{D}}=\mathbb{D} \cup \mathbb{T}$ the closed unit disk. A linear filter with rational transfer function

$$
H(z)=\frac{A\left(z_{1}, \ldots, z_{N}\right)}{P\left(z_{1}, \ldots, z_{N}\right)}
$$

and no nonessential singularities of the second kind is BIBO stable if $P$ has no zeros in the closed unit polydisk $\overline{\mathbb{D}}^{N}$.

A 2-D criterion for testing stability was given by Huang and further developed for the multidimensional case by Anderson and Jury and by Strintzis (see [5] and [10] for more details). Several methods for testing the multidimensional stability where proposed (see [5] and [12, Ch. 3] for an overwiew). More recent developments were obtained in [3], [7]-[9], and [22]. The common background is to characterize BIBO stability throughout a set of $N-1$ positivity conditions, parameterized respectively over $\mathbb{T}^{N-1}, \mathbb{T}^{N-2}, \ldots, \mathbb{\mathbb { T }}$.

The one-dimensional Schur-Cohn stability test relies on Schur coefficients associated to rational functions, which are a powerful tool used in many fields of applications [13]. A widely used framework regards the Cholesky factorization for

Manuscript received July 14, 2006; revised January 9, 2007. The associate editor coordinating the review of this manuscript and approving it for publication was Prof. Brian L. Evans.

The authors are with the Department of Control and Signal Processing, IMS, University Bordeaux, F33405 Talence, France.

Digital Object Identifier 10.1109/TSP.2007.896070 autocorrelation matrices, where the Schur coefficients appear in an equivalent form known as the reflection coefficients, computed via the Levinson algorithm. In the multidimensional case, this equivalence is not longer true.

On one hand, in [14] Liu and Najim proposed a 2-D representation based on the 2-D reflection coefficients and a fast recursive algorithm for their estimation. In [1], the authors tried to extend the Schur-Cohn stability condition in the bidimensional case, using these 2-D reflection coefficients. They obtained a necessary but not sufficient condition of stability. On the other hand, in [20] an analytic extension of the Schur coefficients associated to a contractive analytic function is given. This approach provides a stronger condition of stability, which is a necessary and sufficient condition of stability for multidimensional linear systems. In [19], an overview of the two extensions of the Schur-Cohn stability test that derive from these extended Schur coefficients is presented.

The functional Schur parameters introduced in [20] are a multivariable analogue of the Schur coefficients. They are associated to contractive several-variable analytic functions by means of the notion of "slice functions", a useful tool in multivariable analysis [17]. These coefficients are used to characterize rational inner functions in polydisk algebra, leading to a stability characterization for $N$-variable polynomials.

In this paper, we make use of this analytic approach in order to derive a Schur-Cohn multidimensional extension in the Jurytable form. The stability criterion obtained via slice functions involves only one positivity condition, parameterized over $\mathbb{T}^{N-1}$, rather than the $N-1$ conditions in the Huang test.

We also show how such types of positivity conditions for trigonometric polynomials with complex coefficients can be reduced, in the two-variable case, to the Sturm algorithm. Such simplifications were developed by several authors [5].

The paper is organized as follows: in Section II we recall the background of the 1-D Schur-Cohn test in connection with the Schur algorithm. In Section III, the multidimensional Schur criterion given in [20] is used in order to derive a new stability test in a Jury-table form. Section IV provides examples and comparisons with other existing methods. Section V analyzes the 2-D case, and a simplification to the Sturm algorithm in the case of a polynomial with complex coefficients is proposed. We also provide in this section several 2-D numerical illustrations.

\section{EQUIVALENT FORMS OF THE SCHUR-COHN ALGORITHM}

We briefly recall in this section the background of the SchurCohn algorithm. Given a polynomial $P(z)=\sum_{k=0}^{n} p_{k} z^{k}$ of 
degree $n$ with complex coefficients, $T_{P}$ denotes the infinite Toeplitz matrix associated to $P$

$$
T_{P}=\left[\begin{array}{cccc}
p_{0} & 0 & 0 & \ldots \\
p_{1} & p_{0} & 0 & \ddots \\
p_{2} & p_{1} & p_{0} & \ddots \\
\vdots & \ddots & \ddots & \ddots
\end{array}\right] .
$$

The Schur-Cohn matrix associated to a pair $(P, Q)$ of polynomials is defined by

$$
\Delta(P, Q)=T_{Q} T_{Q}^{H}-T_{P} T_{P}^{H}
$$

and $\Delta_{k}(P, Q)$ denotes the $k \times k$ leading principal submatrix of $\Delta(P, Q)$.

An important particular case of a polynomial pair is $\left(P, P^{\#}\right)$, where $P^{\#}$ is the transpose of $P$, defined by

$$
P^{\#}(z)=z^{n} \bar{P}(1 / \bar{z})=\sum_{k=0}^{n} \bar{p}_{n-k} z^{k} .
$$

In this case, all the entries of the matrix $\Delta\left(P, P^{\#}\right)$ are zero outside $\Delta_{n}\left(P, P^{\#}\right)$, and this latter submatrix is known as the $n \times n$ Schur-Cohn matrix associated to $P$.

The Schur-Cohn criterion can be stated as follows [15]: the polynomial $P$ has all the roots inside the unit disk if and only if the matrix $\Delta_{n}\left(P, P^{\#}\right)$ is positive definite, which amounts to

$$
\operatorname{det} \Delta_{k}\left(P, P^{\#}\right)>0 \quad(k=1,2, \ldots, n) .
$$

This connection between the roots of $P$ and the leading minors of the Schur-Cohn matrix goes through the Schur recursion and the so called Schur coefficients i.e., given a pair $(P, Q)$ of polynomials, one can construct a sequence $\left(P_{k}, Q_{k}\right)_{k=0,1, \ldots}$ of pairs of polynomials defined in the following way: $P_{0}=$ $P, Q_{0}=Q$ and

$$
\begin{aligned}
& P_{k}(z)=\frac{1}{z}\left(P_{k-1}(z)-\gamma_{k-1} Q_{k-1}(z)\right) \quad(k \geq 1) \\
& \left.Q_{k}(z)=Q_{k-1}(z)-\bar{\gamma}_{k-1} P_{k-1}(z)\right) \quad(k \geq 1)
\end{aligned}
$$

where

$$
\gamma_{k}=P_{k}(0) / Q_{k}(0) \quad(k \geq 0)
$$

are called the Schur coefficients of the pair $(P, Q)$. The connection with the Schur-Cohn matrix is the following relation:

$$
\begin{aligned}
\operatorname{det} \Delta_{k}(P, Q) & =\left|Q_{k-1}(0)\right|^{2}-\left|P_{k-1}(0)\right|^{2} \\
& =\left(1-\left|\gamma_{k-1}\right|^{2}\right)\left|Q_{k-1}(0)\right|^{2}
\end{aligned}
$$

for $k \geq 1$. In particular, the positivity condition (4) holds if and only if the Schur coefficients $\left(\gamma_{k}\right)_{k=0, \ldots, n-1}$ of the pair $\left(P, P^{\#}\right)$ satisfy

$$
\left|\gamma_{k}\right|<1 \quad(k=0, \ldots, n-1)
$$

Now the equivalence between (9) and the fact that $P$ has all the roots in the unit disk can be proved by writing the Schur recursion (5), (6) in an equivalent way which emphasizes the quotients $F_{k}:=P_{k} / Q_{k}$ rather than the pairs $\left(P_{k}, Q_{k}\right)$.

Consider the transform $\Phi$ that maps a complex function $F$ analytic around the origin to the function $\Phi(F)$ defined by $\Phi(F)=$ 0 if $F$ is a constant unimodular function, and

$$
\Phi(F)(z)= \begin{cases}\frac{F(z)-F(0)}{z(1-\overline{F(0)} F(z))} & z \neq 0 \\ F^{\prime}(0)\left(1-|F(0)|^{2}\right)^{-1} & z=0\end{cases}
$$

otherwise [4].

Starting with any function $F$, one can construct the sequence of functions $\left(F_{k}\right)_{k=0,1, \ldots}$ using the recursion

$$
F_{0}=F, \quad F_{k}=\Phi\left(F_{k-1}\right) \quad(k \geq 1) .
$$

The parameters

$$
\gamma_{k}=F_{k}(0) \quad(k \geq 0)
$$

are called the Schur coefficients of the function F, and they characterize the function in the sense that a different sequence of Schur coefficients is associated to each function. A straight computation shows that if $F=P / Q$, then $F_{k}=P_{k} / Q_{k}$ for $k \geq 1$, where $\left(P_{k}, Q_{k}\right)$ are defined by (5) and (6). Therefore, (11), (5) and (6) define the same Schur recursion, and the Schur coefficients defined, respectively, in (7) and (12) coincide.

The Schur class $S$ is by definition the closed unit ball of $H^{\infty}(\mathbb{D}):=\left\{f \in H^{\infty}(\mathbb{D}):|f(z)| \leq 1, z \in \mathbb{D}\right\}$. Schur proved in [18], using a simple argument given by the Schwartz lemma, that if $F$ is in the Schur class, then $|\Phi(F)|$ is also bounded by 1. This means that whenever $|F(z)| \leq 1$ for $z \in \mathbb{D}$, the Schur coefficients (12) of $F$ necessarily verify

$$
\left|\gamma_{k}\right| \leq 1 \quad(k \geq 1)
$$

Moreover, equality in (13) can occur for at most one $k$, since $\left|F_{k}(0)\right|=1$ implies, by the maximum modulus principle, that $F_{k}(z)=F_{k}(0)$ for $z$ in $\mathbb{D}$. Therefore, $F_{k}$ is a constant unimodular function and so $F_{k+l}=0$ for $l \geq 1$ (by the definition of $\Phi$ ).

Consider now a polynomial $P$ of degree $n$ and let $F=P / P^{\#}$. If $P(z)=\prod_{j=1}^{n}\left(z-\alpha_{j}\right)$ is the root factorization of $P$, then

$$
F(z)=\prod_{j=1}^{n} \frac{z-\alpha_{j}}{1-\bar{\alpha}_{j} z}
$$

We recall that in digital filtering an all pass filter is a Blaschke product of degree $n$, defined by

$$
B(z)=\prod_{i=1}^{n} \frac{z-b_{i}}{1-\bar{b}_{i} z}, \quad\left(b_{i} \in \mathbb{D}\right) .
$$

The fact that all the roots $\alpha_{j}$ of $P$ lie in the open unit disk is equivalent to the fact that $F$ is a Blaschke product of degree $n$. This means that $F$ is bounded by 1 in $\mathbb{D}$ and that $\left|\gamma_{n}\right|=1$. But 
this happens, as explained earlier, if and only if $\left|\gamma_{k}\right|<1$ for $k=0,1, \ldots, n-1$, i.e., (9).

The equivalent stability condition for $P$ to have all the roots outside the closed unit disk is then simply obtained by switching the roles of $P$ and $P^{\#}$. The Jury table for instance [11] is a way to write the recursion given by (5) and (6) for the pair $\left(P^{\#}, P\right)$ and test positivity of $\operatorname{det} \Delta_{k}(P, Q)$ in (8).

The Jury Table Form of the Schur-Cohn Algorithm

1. Take the polynomial $P(z)=p_{0}+p_{1} z+p_{2} z^{2}+\cdots+p_{n} z^{n}$.

2. Test if $\left|p_{0}\right|^{2}-\left|p_{n}\right|^{2}>0$.

3. Form the coefficients $p_{k}^{1}=\bar{p}_{0} p_{k}-p_{n} \bar{p}_{n-k}$.

4. Restart with $P_{1}(z)=p_{0}^{1}+p_{1}^{1} z+\cdots+p_{n-1}^{1} z^{n-1}$ instead of $P$.

\section{Functional Schur CoEFFicients AND EXTENDED SCHUR-COHN CRITERION}

In [20], the definition of the Schur coefficients sequence for an analytic function $F$ is extended to analytic functions in several variables, by means of the so-called "slice functions" [17].

Let $F$ be an analytic function in the open unit polydisk $\mathbb{D}^{N}$. For each point $w=\left(w_{1}, \ldots, w_{N}\right)$ on the polytorus $\mathbb{T}^{N}$ let $D_{w}$ be the one-dimensional disk that "slices" $\mathbb{D}^{N}$ through the origin and through $w$

$$
D_{w}=\left\{\lambda w=\left(\lambda w_{1}, \ldots, \lambda w_{N}\right): \lambda \in \mathbb{D}\right\} .
$$

Consider the restriction of $F$ to the one-dimensional disk $D_{w}$, which can be regarded as a one variable function

$$
F_{w}(\lambda)=F(\lambda w) \quad(\lambda \in \mathbb{D}) .
$$

$F_{w}$ is called the slice of $F$ through $w$ [17]. For each $w$ on $\mathbb{T}^{N}$ define, by the Schur recursion (11), the sequence $\left(F_{w, k}\right)_{k \geq 0}$

$$
F_{w, 0}=F_{w} \quad F_{w, k}=\Phi\left(F_{w, k-1}\right) \quad(k \geq 1) .
$$

The functions $\gamma_{k}: \mathbb{T}^{N} \rightarrow \mathbb{C}$ defined in [20] by

$$
\gamma_{k}(w)=F_{w, k}(0) \quad\left(w \in \mathbb{T}^{N}\right)
$$

are called the functional Schur coefficients of the function F.

In order to write the transpose of a polynomial in the multivariable case we will introduce some notations. For a multiindex $\alpha=\left(\alpha_{1}, \ldots, \alpha_{N}\right) \in \mathbb{N}^{N}$ and for $z=\left(z_{1}, \ldots z_{N}\right) \in \mathbb{C}^{N}$ the multi-index notation $z^{\alpha}$ stands for the monomial $z_{1}^{\alpha_{1}} \ldots z_{N}^{\alpha_{N}}$ whose degree is $|\alpha|=\alpha_{1}+\cdots+\alpha_{N}$.

If

$$
P(z)=\sum_{|\alpha| \leq n} p_{\alpha} z^{\alpha}
$$

is a polynomial in $N$ variables of degree $n$, one can define the transpose polynomial $P^{\#}$ as

$$
P^{\#}(z)=M_{0}(z) \bar{P}\left(\frac{1}{z}\right)
$$

where $M_{0}$ is the monomial of the least degree such that $M_{0}(z) \bar{P}(1 / z)$ is a polynomial, and

$$
\frac{1}{z}=\left(\frac{1}{z_{1}}, \ldots, \frac{1}{z_{N}}\right) .
$$

More explicitly, define $\beta=\left(\beta_{1}, \ldots, \beta_{N}\right) \in \mathbb{N}^{N}$ by

$$
\beta_{i}=\max _{|\alpha| \leq n, p_{\alpha} \neq 0} \alpha_{i} \quad(i=1, \ldots, N)
$$

which represent the dimensions of the smallest $N$-dimensional rectangular "box" in $\mathbb{N}_{+}^{N}$ with one corner at the origin and containing the multiindexes of all the coefficients of $P$ different from zero. Then the transpose $P^{\#}$ is the polynomial of degree $|\beta|$

$$
P^{\#}(z)=\sum_{|\alpha| \leq|\beta|} \bar{p}_{\beta-\alpha} z^{\alpha} .
$$

It is shown in [20] that for any $N$-variable analytic function $F$ and for each $k \geq 1$, the functional Schur coefficient $\gamma_{k}$ is a continuous function in every point $w$ of $\mathbb{T}^{N}$ for which the slice $F_{w}$ is not a Blaschke product of degree less than or equal to $k$. This is a consequence of the fact that the correspondence $w \mapsto F_{w}$ is weakly continuous. The particular case of most interest is, as in the 1-D case, when $F$ is written as $P^{\#} / P$ for some $N$-variable polynomial $P$. The properties of these rational inner functions [17] are used in [20] in conjunction with the continuity property aforementioned in order to show the following equivalent conditions relating the stability to the functional Schur coefficients.

Theorem 3.1: Let $P$ be a polynomial of degree $n$ in $N$ variables, $F(z)=\left(P^{\#}(z) / P(z)\right)$ and $\left(\gamma_{k}\right)_{k \in \mathbb{N}}$ be the sequence of functional Schur coefficients of $F$. Denote $n^{\#}=\operatorname{deg}\left(P^{\#}\right)$. The following statements are equivalent.

a) The polynomial $P$ has no zeros in the closed unit polydisk $\overline{\mathbb{D}^{n}}$

b) $\gamma_{k}$ are continuous in each $w$ for each $k \geq 0$;

c) $F$ belongs to the disk algebra $A\left(\mathbb{D}^{N}\right)$ (i.e., $F$ is continuous on $\overline{\mathbb{D}^{n}}$;

d) $F_{w}$ is a Blaschke product of degree $n^{\#}$ for all $w \in \mathbb{T}^{N}$;

e) For each $w \in \mathbb{T}^{N}$ we have

$$
\begin{aligned}
\left|\gamma_{n^{\#}}(w)\right| & =1 \\
\left|\gamma_{k}(w)\right| & <1 \quad\left(0 \leq k \leq n^{\#}-1\right) .
\end{aligned}
$$

These equivalent conditions can be simplified using two independent arguments.

1) Take $\beta$ as in (19), so $|\beta|=n^{\#}$, and put $q=n^{\#}-n$. A simple computation shows the following relationship between slicing and transposing:

$\left(P^{\#}\right)_{w}(\lambda)=w^{\beta} \lambda^{q}\left(P_{w}\right)^{\#}(\lambda) \quad\left(w \in \mathbb{T}^{n}, \lambda \in \mathbb{D}\right)$

and, therefore

$\left(\frac{P^{\#}}{P}\right)_{w}(\lambda)=w^{\beta} \lambda^{q} \frac{\left(P_{w}\right)^{\#}(\lambda)}{P_{w}(\lambda)} \quad\left(w \in \mathbb{T}^{n}, \lambda \in \mathbb{D}\right)$.

The last relationship shows that the condition d) in the Theorem 3.1 is equivalent to the fact that $\left(P_{w}\right)^{\#} / P_{w}$ is a 
Blaschke product of degree $n$ for all $w \in \mathbb{T}^{N}$. Thus, in the second relation of e) one might replace the first $n^{\#}$ functional Schur coefficients of $P^{\#} / P$ with the first $n$ Schur coefficients of $\left(P_{w}\right)^{\#} / P_{w}$.

2) One does not have to consider all the slices through the points $w$ on $\mathbb{T}^{N}$, because many of them are "redundant." More precisely, if $w$ and $w^{\prime}$ are two points on $\mathbb{T}^{N}$ such that $w^{\prime}=u w$ for some $u \in \mathbb{T}$, then the disks $D_{w}$ and $D_{w^{\prime}}$ in (16) coincide. One coordinate can then be "normalized," say the last one

$$
\left(w_{1}, \ldots, w_{N}\right)=w_{N}\left(w_{1} / w_{N}, \ldots, w_{N-1} / w_{N}, 1\right)
$$

so one can consider only the disks of the type

$$
D_{v}=\left\{\lambda\left(v_{1}, \ldots, v_{N-1}, 1\right): \lambda \in \mathbb{D}\right\} \quad\left(v \in \mathbb{T}^{N-1}\right)
$$

and the corresponding "normalized" Schur coefficients denoted $\left(\tilde{\gamma}_{k}\right)_{k \geq 0}$, defined as in (18) but only for the slicing disks (25), i.e., as functions of $v \in \mathbb{T}^{N-1}$.

Summing up, and generalizing the Schur-Cohn criterion written as in (9), the following multidimensional Schur-Cohn criterion is obtained.

Corollary 3.2: Let $P$ be a polynomial in $N$ variables of degree $n$. For $v \in \mathbb{T}^{N-1}$, let $\left(\tilde{\gamma}_{k}(v)\right)_{0 \leq k \leq n-1}$ be the first $n$ Schur coefficients of the one-variable function

$$
\tilde{F}_{v}(\lambda)=\frac{\left(P\left(\lambda v_{1}, \ldots \lambda v_{N-1}, \lambda\right)\right)^{\#}}{P\left(\lambda v_{1}, \ldots \lambda v_{N-1}, \lambda\right)} .
$$

The following statements are equivalent:

A) $P$ has no zeros in the closed unit polydisk $\overline{\mathbb{D}}^{N}$;

B) $P_{v}(\lambda)=P\left(\lambda v_{1}, \ldots \lambda v_{N-1}, \lambda\right)$ has no zeros in $\overline{\mathbb{D}}$ for $v \in \mathbb{T}^{N-1}$;

C) $\left|\tilde{\gamma}_{k}(v)\right|<1$ for $v \in \mathbb{T}^{N-1}$ and $0 \leq k \leq n-1$.

The stability condition $C$ ) in the last corollary can be written in Jury table form by developing the slice expansions in (26) and using positivity in (8) instead of (9). If $P(z)=\sum_{|\alpha| \leq n} p_{\alpha} z^{\alpha}$ and $v=\left(v_{1}, \ldots, v_{N-1}\right) \in \mathbb{T}^{N-1}$, then

$$
P_{v}(\lambda)=\sum_{k=1}^{n} c_{k}(v) \lambda^{k} \quad(\lambda \in \mathbb{D})
$$

where the coefficients $c_{k}$ are polynomials in $v$ given by

$$
c_{k}(v)=\sum_{|\alpha|=k} p_{\alpha} v_{1}^{\alpha_{1}} v_{2}^{\alpha_{1}} \ldots v_{N-1}^{\alpha_{N-1}} \quad(0 \leq k \leq n) .
$$

The extended algorithm then goes through the following steps:

The Jury Table Form of the n-D Slice-Based Schur-Cohn Algorithm

1) Take $P$ and form the polynomials $c_{k}$, for $k=0, \ldots, n$.

2) Test if the trigonometric polynomial

$$
\Delta(v)=\left|c_{0}(v)\right|^{2}-\left|c_{n}(v)\right|^{2}
$$

is positive for $v \in \mathbb{T}^{N-1}$.
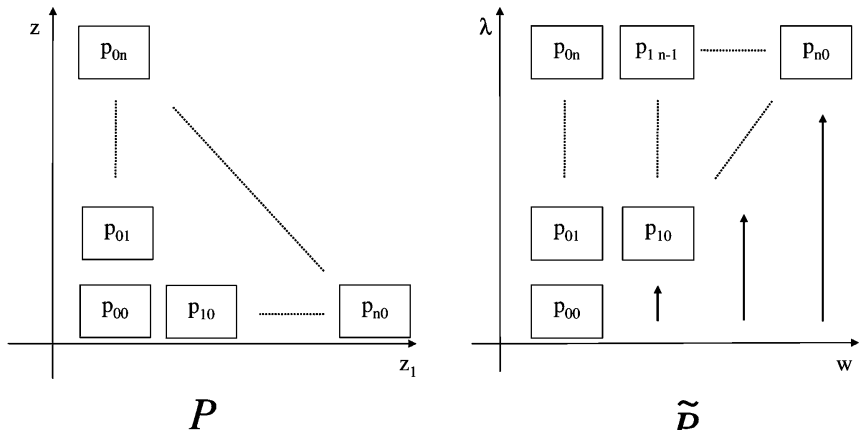

Fig. 1. Shifting the coefficients.

3) Form the new polynomial coefficients

$$
\begin{array}{r}
c_{k}^{1}(v)=\bar{c}_{0}(v) c_{k}(v)-c_{n}(v) \bar{c}_{n-k}(v) \\
(0 \leq k \leq n-1) .
\end{array}
$$

4) Restart with the sequence $\left(c_{k}^{1}\right)_{0 \leq k \leq n-1}$ instead of $\left(c_{k}\right)_{0 \leq k \leq n}$.

Alternatively, one can derive a similar algorithm based on the Bistritz table [2] instead of the Jury table. The use of Jury table can better emphasize the connection between the Schur coefficients and the leading minors in the Schur-Cohn matrix, while the use of Bistritz table reduces the computational complexity.

\section{COMPARISONS AND EXAMPLES}

It is natural to compare condition B) in Corollary 3.2 with the Huang-Jury-Anderson stability conditions which we state here for the convenience of the reader: $P$ has no zeros in $\overline{\mathbb{Z}}^{N}$ if and only if the following conditions hold for $\lambda \in \mathbb{Z}$ :

$\left(C_{N-1}\right): P\left(v_{1}, \ldots, v_{N-1}, \lambda\right) \neq 0 \quad\left(\left(v_{1}, \ldots, v_{N-1}\right) \in \mathbb{T}^{N-1}\right)$

$\left(C_{N-2}\right): P\left(v_{1}, \ldots, v_{N-2}, \lambda, 0\right) \neq 0 \quad\left(\left(v_{1}, \ldots, v_{N-2}\right) \in \mathbb{T}^{N-2}\right)$

...

$\left(C_{1}\right): P\left(v_{1}, \lambda, 0, \ldots, 0\right) \neq 0 \quad\left(v_{1} \in \mathbb{T}\right)$

$\left(C_{0}\right): P(\lambda, 0, \ldots, 0) \neq 0$.

It follows from (27) and (28) that, for $\lambda \in \mathbb{Z}$ and $v=\left(v_{1}, \ldots, v_{N-1}\right) \in \mathbb{T}^{N-1}$

$$
P_{v}(\lambda)=\tilde{P}\left(v_{1}, \ldots, v_{N-1}, \lambda\right)
$$

where the polynomial $\tilde{P}(z)=\sum_{\alpha} \tilde{p}_{\alpha} z^{\alpha}$ is obtained from $P$ by "shifting up" the coefficients of $P$ along the last coordinate: (see Fig. 1)

$$
\tilde{p}_{\left(\alpha_{1}, \ldots, \alpha_{N-1}, \alpha_{1}+\cdots+\alpha_{N-1}\right)}=p_{\left(\alpha_{1}, \ldots, \alpha_{N}\right)} \quad\left(\alpha \in \mathbb{N}^{N}\right) .
$$

Thus, (30) shows that the slice-based condition B) in the Corollary 3.2 is equivalent to only the first condition $\left(C_{N-1}\right)$, applied for $\tilde{P}$ instead of $P$, so by using B) one has to check only one condition instead of $N$. A counterpart is that the degree of $\tilde{P}$ exceeds $n$ (it is no greater than $2 n$ ), so numerically it is more 
efficient to use 3.2 B) for polynomials of small degree in many variables.

Since the normalization (25) can be done in any of the $N$ variables, the normalization variable can be chosen in order to minimize the degree of $\tilde{P}$ in the range $[n, 2 n]$. More precisely, if $\beta=\left(\beta_{1}, \ldots, \beta_{N}\right)$ are, as in (19), the dimensions of the minimal $N$-dimensional "box" containing the coefficients indexes for $P$, then it follows from (31) that the minimal degree of $\tilde{P}$ is attained when normalizing $P$ in the variable $z_{i_{0}}$ such that $\beta_{i_{0}}$ is the minimum of $\beta_{1}, \ldots, \beta_{N}$.

Let us illustrate by some comparative examples the simplifications provided by the proposed method.

Example 1: Consider the following polynomial:

$$
P_{1}\left(z_{1}, z_{2}\right)=1+\frac{1}{2} z_{1}+\frac{1}{2} z_{2}+\frac{1}{4} z_{1} z_{2}+\frac{1}{4} z_{1}^{2}+\frac{1}{4} z_{2}^{2} .
$$

I. Checking the stability of $P_{1}$ for instance with the method in [9] requires the following conditions to be satisfied:

1) $p_{0}\left(z_{1}\right)$ has no zeros on the unit circle;

2) The following matrix function $E\left(z_{1}\right)$ is positive definite on the unit circle:

$$
\begin{aligned}
E\left(z_{1}\right) & =\left(\begin{array}{cc}
p_{0}\left(z_{1}\right) & 0 \\
p_{1}\left(z_{1}\right) & p_{0}\left(z_{1}\right)
\end{array}\right)\left(\begin{array}{cc}
\overline{p_{0}}\left(\frac{1}{z_{1}}\right) & \overline{p_{1}}\left(\frac{1}{z_{1}}\right) \\
0 & \overline{p_{0}}\left(\frac{1}{z_{1}}\right)
\end{array}\right) \\
& -\left(\begin{array}{cc}
\overline{p_{2}}\left(\frac{1}{z_{1}}\right) & 0 \\
\overline{p_{1}}\left(\frac{1}{z_{1}}\right) & \overline{p_{2}}\left(\frac{1}{z_{1}}\right)
\end{array}\right)\left(\begin{array}{cc}
p_{2}\left(z_{1}\right) & p_{1}\left(z_{1}\right) \\
0 & p_{2}\left(z_{1}\right)
\end{array}\right)
\end{aligned}
$$

where

$$
\begin{aligned}
& p_{0}\left(z_{1}\right)=1+\frac{1}{2} z_{1}+\frac{1}{4} z_{1}^{2} \\
& p_{1}\left(z_{1}\right)=\frac{1}{2}+\frac{1}{4} z_{1} \text { and } p_{2}\left(z_{1}\right)=\frac{1}{4} .
\end{aligned}
$$

Note that the matrix $E$ is in fact the Schur-Cohn matrix associated to $P_{1}\left(\cdot, z_{2}\right)$ as shown in Section 1 . The positivity of $E\left(z_{1}\right)$ is equivalent with the positivity of the entry $(1,1)$ of the matrix and the positivity of the determinant of $E_{1}$. Summing up, to verify the stability of $P_{1}$ the following conditions must be tested :

$$
\begin{aligned}
& 1+\frac{1}{2} z_{1}+\frac{1}{4} z_{1}^{2} \neq 0, \quad\left|z_{1}\right| \leq 1 \\
& \Delta_{1}\left(z_{1}\right)=\frac{5}{4}+\frac{5}{8}\left(z_{1}+z_{1}^{-1}\right) \\
& \quad+\frac{1}{4}\left(z_{1}^{2}+z_{1}^{-2}\right)>0, \quad\left|z_{1}\right|=1 \\
& \Delta_{2}\left(z_{1}\right)=\operatorname{det} E\left(z_{1}\right) \\
& \quad=133+102\left(z_{1}+z_{1}^{-1}\right)+57\left(z_{1}^{2}+z_{1}^{-2}\right) \\
& \quad+18\left(z_{1}^{3}+z_{1}^{-3}\right)+4\left(z_{1}^{4}+z_{1}^{-4}\right)>0, \quad\left|z_{1}\right|=1 .
\end{aligned}
$$

II. On the other hand, by the proposed criterion of stability 3.2 , the polynomial $P_{1}$ has no zeros inside the closed unit polydisk if and only if $P_{w}(\lambda) \neq 0$ in the closed unit polydisk, where

$$
P_{w}(\lambda)=1+\frac{1}{2} \lambda(w+1)+\frac{1}{4} \lambda^{2}\left(w^{2}+w+1\right) .
$$

This is equivalent to the positivity on the unit circle of the matrix function $E(w)$, constructed as in (32), where

$$
\begin{aligned}
& p_{0}(w)=1, p_{1}(w)=\frac{1}{2}(1+w) \\
& p_{2}(w)=\frac{1}{4}\left(1+w+w^{2}\right) .
\end{aligned}
$$

Furthermore, $E$ is positive definite if and only if the following inequalities hold for $|w|=1$ :

$$
\begin{aligned}
\Delta_{1}(w)= & \frac{13}{16}-\frac{1}{8}\left(w+w^{-1}\right)-\frac{1}{16}\left(w^{2}+w^{-2}\right)>0 \\
\Delta_{2}(w)= & \operatorname{det} E_{1}\left(z_{1}\right)=\frac{1}{256}\left[139-48\left(w+w^{-1}\right)\right. \\
& \left.-6\left(w^{2}+w^{-2}\right)+\left(w^{4}+w^{-4}\right)\right]>0 .
\end{aligned}
$$

In conclusion, (33), (34), and (35) can be replaced with (36) and (37), resulting in a lower overall computational cost. The difference between the computational cost of the methods increases with the number of variables, as illustrated in the following 3-D example of stable polynomial.

Example 2: Consider the polynomial

$$
\begin{array}{r}
P_{2}\left(z_{1}, z_{2}, z_{3}\right)=5+0.25 z_{1}+0.25 z_{2} \\
+0.25 z_{3}+z_{1}^{2}+z_{2}^{2}+z_{3}^{2} .
\end{array}
$$

I. By the Anderson-Jury criterion of stability, $P_{2}$ is stable if and only if

$$
\begin{gathered}
P_{2}\left(z_{1}, 0,0\right)=5+.25 z_{1}+z_{1}^{2} \neq 0, \quad\left|z_{1}\right| \leq 1 \\
P_{2}\left(z_{1}, z_{2}, 0\right)=5+.25 z_{1}+.25 z_{2}+z_{1}^{2}+z_{2}^{2} \neq 0 \\
\left|z_{1}\right|=1,\left|z_{2}\right| \leq 1 \\
P_{2}\left(z_{1}, z_{2}, z_{3}\right)= \\
+z_{1}+.25 z_{1}+.25 z_{2}+.25 z_{3} \\
+z_{2}^{2}+z_{3}^{2} \neq 0 \\
\left|z_{1}\right|=\left|z_{2}\right|=1,\left|z_{3}\right| \leq 1
\end{gathered}
$$

The condition (39) is equivalent with the positivity of $\Delta_{1}\left(z_{1}\right)$ and of $\Delta_{2}\left(z_{1}\right)$ on the circle, where

$$
\begin{aligned}
\Delta_{1}\left(z_{1}\right)= & 25.0625+1.5\left(z_{1}+z_{1}^{-1}\right)+5\left(z_{1}^{2}+z_{1}^{-2}\right) \\
\Delta_{2}\left(z_{1}\right)= & 681.5625+90.109375\left(z_{1}+z_{1}^{-1}\right) \\
& +252.625\left(z_{1}^{2}+z_{1}^{-2}\right) \\
& +15\left(z_{1}^{3}+z_{1}^{-3}\right)+25\left(z_{1}^{3}+z_{1}^{-3}\right) .
\end{aligned}
$$

Condition (40) is equivalent with the positivity of $\Delta_{1}\left(z_{1}, z_{2}\right)$ and of $\Delta_{2}\left(z_{1}, z_{2}\right)$ for $\left|z_{1}\right|=\left|z_{2}\right|=1$, where

$$
\begin{aligned}
\Delta_{1}\left(z_{1}, z_{2}\right) & =\left|5+0.25\left(z_{1}+z_{2}\right)+0.5\left(z_{1}^{2}+z_{2}^{2}\right)\right|^{2}-1 \\
b\left(z_{1}, z_{2}\right) & =1+0.0625\left(z_{1}+z_{2}\right)+.25\left(z_{1}^{2}+z_{2}^{2}\right) \\
\Delta_{2}\left(z_{1}, z_{2}\right) & =\left|\Delta_{1}\left(z_{1}, z_{2}\right)\right|^{2}-\left|b\left(z_{1}, z_{2}\right)\right|^{2} .
\end{aligned}
$$

Thus, a total of five conditions are to be verified, i.e., (38) and (41) to (44).

II. When testing the stability of $P_{2}$ with the proposed criterion, one has to check

$P_{w}(\lambda)=\lambda^{2}\left(1++w_{1}^{2}+w_{2}^{2}\right)+0.25 \lambda\left(w_{1}+w_{2}+1\right)+5 \neq 0$ 

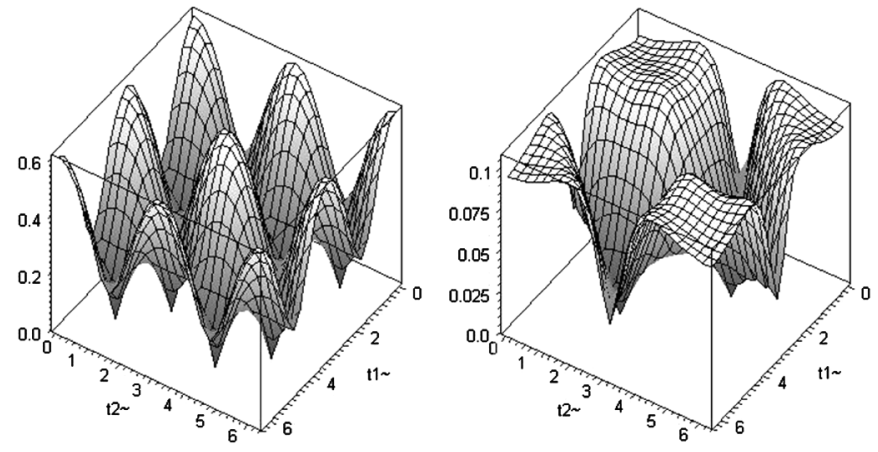

Fig. 2. $\left|\gamma_{1}\left(w_{1}, w_{2}\right)\right|$ and $\left|\gamma_{2}\left(w_{1}, w_{2}\right)\right|$ for $P_{2}$.

for $\left|w_{1}\right|=\left|w_{2}\right|=1$. This condition is similar to condition (40), and is equivalent with the positivity of $\Delta_{1}\left(w_{1}, w_{2}\right)$ and of $\Delta_{2}\left(w_{1}, w_{2}\right)$ for $\left|w_{1}\right|=\left|w_{2}\right|=1$, where

$$
\begin{aligned}
\Delta_{1}\left(w_{1}, w_{2}\right)= & 25-\left|1+w_{1}^{2}+w_{2}^{2}\right|^{2} \\
b\left(w_{1}, w_{2}\right)= & 1+w_{1}^{-1}+w_{2}^{-1}-.25\left(w_{2}+w_{1}\right) \\
& -.25\left(w_{1}^{-2} w_{2}+w_{1} w_{2}^{-2}\right)-.25\left(w_{1}^{2}+w_{2}^{2}\right) \\
\Delta_{2}\left(w_{1}, w_{2}\right)= & \left|\Delta_{1}\left(w_{1}, w_{2}\right)\right|^{2}-\left|b\left(w_{1}, w_{2}\right)\right|^{2} .
\end{aligned}
$$

Therefore, when using the proposed n-D Schur-Cohn criterion two conditions are needed, i.e., (45) and (46) instead of five. Note that the positivity of $\Delta_{1}\left(w_{1}, w_{2}\right)$ and of $\Delta_{2}\left(w_{1}, w_{2}\right)$ is equivalent with $\left|\gamma_{1}\left(w_{1}, w_{2}\right)\right|<1$ and $\left|\gamma_{2}\left(w_{1}, w_{2}\right)\right|<1$, where $\gamma_{i}$ are the functional Schur coefficients for $P_{2}$ (see Fig. 2).

\section{A SimplificAtion to THE STURM AlgORIthM FOR TWO-VARIABLE POLYNOMIALS}

As shown in the previous section, testing the stability of an $N$-variable polynomial $P$, either with Huang-type conditions or with slice-based conditions, goes through testing the positivity on a several-dimension polytorus of some real valued trigonometric polynomials $\Delta$ with complex coefficients, such as in (29).

In the particular case of a two-variable polynomial $P$ with complex coefficients, this comes down to checking if some trigonometric polynomials of the type

$$
\Delta(v)=\sum_{k=-m}^{m} \delta_{k} v^{k} \quad(v \in \mathbb{T})
$$

with $\delta_{-k}=\bar{\delta}_{k}$, are positive on the unit circle.

A common way to numerically check such positivity conditions is to use a change of variable in $\Delta$, in order to reduce it to a real variable polynomial with real coefficients. Several methods can be then used to check the positivity of the reduced polynomial. This can be done either directly, with Sturm type algorithms [21], or by checking if the polynomial can be written as a sum of squares such in [16], which is more efficient in terms of complexity. Remark that [16] can also deal with polynomials of two variables and that in this case the generalized Sturm theorem can be used (see [5, Ch. 2] for more details).
If $\Delta$ has real coefficients, then it can be written as

$$
\Delta(v)=\sum_{k=0}^{m} \delta_{k}\left(v^{k}+\bar{v}^{k}\right)
$$

In this case a classical change of variables is used [5, Sec. 3.4]

$$
t=\frac{1}{2}(v+\bar{v})=2 \operatorname{Re} v=\cos \theta \quad\left(v=e^{i \theta} \in \mathbb{T}\right) .
$$

The polynomial $\Delta$ then becomes

$$
\Delta(t)=\sum_{k=0}^{m} \delta_{k} \cos (k \theta)=\sum_{k=0}^{m} \delta_{k} T_{k}(t) \quad(t \in[-1,1])
$$

where $\left(T_{k}\right)_{0 \leq k \leq m}$ are the Chebyshev polynomials of the first kind.

However, (48) is no longer true in the general case of polynomials $\Delta$ with complex coefficients. The stability of polynomials with complex coefficients can be then tested by multiplying the given polynomial with the polynomial obtained by complex conjugation of its coefficients [5], [7].

We propose in this paper a different approach using a change of variable based on the omographic transform $\psi$

$$
\psi(z)=\frac{z-i}{z+i} \quad(z \in \mathbb{C})
$$

which maps the upper half-plane onto the open unit disk and the real line $\mathbb{R}$ onto $\mathbb{T} \backslash\{1\}$.

More precisely, in (48) let

$$
v:=\frac{t-i}{t+i} \quad(t \in \mathbb{R}) .
$$

Let us define

$$
Q(t)=\frac{\left(t^{2}+1\right)^{m}}{2} \Delta(\psi(t)) \quad(t \in \mathbb{R}) .
$$

Since

$$
Q(t)=\frac{\left(t^{2}+1\right)^{m}}{2} \sum_{k=-m}^{m} \delta_{k} \frac{(t-i)^{k}}{(t+i)^{k}} \quad(t \in \mathbb{R})
$$

one can see that $Q$ is a polynomial in $t$ of degree at most $2 \mathrm{~m}$, say

$$
Q(t)=\sum_{l=0}^{2 m} q_{l} t^{l}
$$

The coefficients $q_{l}$ of $Q$ are real and can be obtained from the coefficients $\delta_{k}$ of $\Delta$ as follows: for $m \geq 1$ consider the (universal) matrices $E^{m}$ and $F^{m}$, of sizes $(m+1) \times(m+1)$ and $m \times m$, respectively

$$
\begin{aligned}
& E^{1}=\left[\begin{array}{cc}
1 & -1 \\
1 & 1
\end{array}\right] \quad F^{1}=[2] \\
& E^{2}=\left[\begin{array}{ccc}
1 & -1 & 1 \\
2 & 0 & -6 \\
1 & 1 & -1
\end{array}\right] \\
& F^{2}=\left[\begin{array}{cc}
2 & -4 \\
2 & 4
\end{array}\right]
\end{aligned}
$$




$$
\begin{aligned}
E^{3} & =\left[\begin{array}{cccc}
1 & -1 & 1 & 1 \\
3 & -1 & 5 & 15 \\
3 & 1 & -5 & -15 \\
1 & 1 & 1 & 1
\end{array}\right] \\
F^{3} & =\left[\begin{array}{ccc}
2 & -4 & 6 \\
4 & 0 & -20 \\
2 & 4 & 6
\end{array}\right]
\end{aligned}
$$

and in general

$$
E_{s, k}^{m}=\sum_{j=\max \{0, k+s-m\}}^{\min \{k, s\}}(-1)^{k-j} C_{m-k}^{s-j} C_{2 k}^{2 j}
$$

for $0 \leq k, s \leq m$, and

$$
F_{s, k}^{m}=\sum_{j=\max \{1, k+s-m\}}^{\min \{k, s\}}(-1)^{k-j} C_{m-k}^{s-j} C_{2 k}^{2 j-1}
$$

for $1 \leq k, s \leq m$. Then the coefficients of $Q$ are given by

$$
\left[\begin{array}{c}
q_{0} \\
q_{2} \\
\vdots \\
q_{2 m}
\end{array}\right]=E^{m}\left[\begin{array}{c}
\delta_{0} / 2 \\
\operatorname{Re} \delta_{1} \\
\vdots \\
\operatorname{Re} \delta_{m}
\end{array}\right]\left[\begin{array}{c}
q_{1} \\
q_{3} \\
\vdots \\
q_{2 m-1}
\end{array}\right]=F^{m}\left[\begin{array}{c}
\Im \delta_{1} \\
\Im \delta_{2} \\
\vdots \\
\Im \delta_{m}
\end{array}\right]
$$

as shown in the Appendix. Therefore, $Q$ is a polynomial of one real variable $t$, with real coefficients and degree not greater than $2 m$. Moreover, it follows from (52) that the trigonometric polynomial $\Delta$ is positive on $\mathbb{T} \backslash\{1\}$ if and only if $Q$ is positive on $\mathbb{R}$, and this can be tested in the classical way with the Sturm algorithm.

The point 1 on the unit circle is an exception and requires specific treatment. Even if $Q$ is positive on $\mathbb{R}$, since

$$
\Delta(1)=\lim _{t \rightarrow \infty} \frac{2 Q(t)}{\left(t^{2}+1\right)^{m}}
$$

it is possible that $\Delta(1)=0$. This clearly happens if and only if the degree of $Q$ is less than $2 m$. Thus one can check either if $q_{2 m}=0$, or directly if $\Delta(1)=0$, case in which the positivity of $\Delta$ fails.

A similar approach uses the omographic transform

$$
\phi(z)=\frac{z+1}{z-1} \quad(z \in \mathbb{C})
$$

carrying the unit circle onto the imaginary axis, and involving the Q-matrix methods [10]. They are suitable for computer implementation and are used in the real coefficient case [6].

Let us present some numerical examples for testing the stability of a 2-D polynomial with real or complex coefficients, using the algorithm presented.

Example 1, Continued: Consider again the polynomial

$$
P_{1}\left(z_{1}, z_{2}\right)=1+\frac{1}{2} z_{1}+\frac{1}{2} z_{2}+\frac{1}{4} z_{1} z_{2}+\frac{1}{4} z_{1}^{2}+\frac{1}{4} z_{2}^{2}
$$

and the principal leading Schur-Cohn minors $\Delta_{1}(w)$ and $\Delta_{2}(w)$ given by (36) and (37).
By the variable change $w:=(t-i / t+i)(t \in \mathbb{R})$ as previously presented in (53), one needs to check that the following polynomials are positive:

$$
\begin{aligned}
q_{1}(t) & =7 t^{4}+38 t^{2}+15=\left(5+t^{2}\right)\left(7 t^{2}+3\right) \\
q_{2}(t) & =225+356 t^{6}+1094 t^{4}+740 t^{2}+33 t^{8} \\
& =\left(33 t^{4}+26 t^{2}+9\right)\left(5+t^{2}\right)^{2} .
\end{aligned}
$$

This is easily checked using for instance the software package [16].

Example 3: Consider the polynomial $P_{3}$ and his slice $P_{w}(\lambda)$

$$
\begin{aligned}
P_{3}\left(z_{1}, z_{2}\right)= & 1+(.2+I) z_{1}++.8 I z_{1}^{2} \\
& +\left(-.5-.5 I+z_{1}\right) z_{2}+\left(1+.5 z_{1}\right) z_{2}^{2} \\
P_{w}(\lambda)= & 1-(.5-.5 I+.2 w+1 I w) \lambda \\
& +\left(1-1 w+.8 I w^{2}\right) \lambda^{2}+.5 \lambda^{3} w .
\end{aligned}
$$

The stability of $P$ is equivalent with the positivity on the unit circle of

$$
\begin{aligned}
\Delta_{1}(w)= & .75 \\
\Delta_{2}(w)= & -1.7625+w+w^{-1}+.45 I\left(w-w^{-1}\right) \\
& -.4\left(w^{-2}+w^{2}\right)+.72 I\left(w^{-2}-w^{2}\right) .
\end{aligned}
$$

Checking the positivity of $\Delta_{2}$ via the change of variable (53) yields to check if

$$
q_{2}(t)=-4.5625+7.56 t+1.275 t^{2}-3.96 t^{3}-.5625 t^{4}
$$

is positive, which is not true, therefore, $P_{3}$ is unstable.

Example 4: This is an example of a stable polynomial

$P\left(z_{1}, z_{2}\right)=1+(.9-.001 I) z_{1}$

$$
+(0.01+0.01 I) z_{2}+.1 I z_{1}^{2} .
$$

The polynomials to be tested for the positivity are $q_{1}(t)=0.99$ and

$$
\begin{aligned}
q_{2}(t)=.14691319 t^{4}+ & 0.0436084 t^{3} \\
& +.32263398 t^{2}+0.0436084 t+.17604079 .
\end{aligned}
$$

\section{CONCLUSION}

In the paper, we have proposed a new multidimensional necessary and sufficient BIBO stability condition based on slicetype extensions of the Schur coefficients. This leads to a new Schur-Cohn type extension, which only needs a unique condition to be checked, as an alternative to the set of $N-1$ conditions of the known algorithms. In the bidimensional case, we have also presented a method to reduce this algorithm to the Sturm algorithm, for polynomials with complex coefficients. Numerical 2-D and 3-D examples where given to illustrate these developments. 


\section{APPENDIX \\ PROOF OF (59)}

For $0 \leq k \leq m$ denote $u_{k}=\operatorname{Re} \delta_{k}$ and $v_{k}=\Im \delta_{k}$. From (53) we have

$$
\begin{aligned}
Q(t) & =\frac{\left(t^{2}+1\right)^{m}}{2} \sum_{k=-m}^{m} \delta_{k}\left(\frac{t-i}{t+i}\right)^{k} \\
& =\frac{\delta_{0}}{2}\left(t^{2}+1\right)^{m}+\sum_{k=1}^{m} X_{k}(t)
\end{aligned}
$$

where

$$
\begin{aligned}
X_{k}(t):= & \frac{1}{2}\left[\delta_{-k}(t+i)^{m+k}(t-i)^{m-k}\right. \\
& \left.\left.+\delta_{k}(t+i)^{m-k}(t-i)^{m+k}\right)\right] \\
= & \frac{\left(t^{2}+1\right)^{m-k}}{2}\left[\delta_{k}(t-i)^{2 k}+\bar{\delta}_{k}(t+i)^{2 k}\right] \\
= & \left(t^{2}+1\right)^{m-k} \operatorname{Re}\left[\delta_{k}(t-i)^{2 k}\right] \\
= & \left(t^{2}+1\right)^{m-k}\left[u_{k} \operatorname{Re}(t-i)^{2 k}-v_{k} \Im(t-i)^{2 k}\right] .
\end{aligned}
$$

Now

$$
\operatorname{Re}(t-i)^{2 k}=\sum_{j=0}^{k} C_{2 k}^{2 j}(-1)^{k-j} t^{2 j}
$$

and

$$
\Im(t-i)^{2 k}=\sum_{j=1}^{k} C_{2 k}^{2 j-1}(-1)^{k-j+1} t^{2 j-1}
$$

so

$$
\begin{aligned}
X_{k}(t)= & \left(\sum_{l=0}^{m-k} C_{m-k}^{l} t^{2 l}\right)\left[u_{k} \sum_{j=0}^{k} C_{2 k}^{2 j}(-1)^{k-j} t^{2 j}\right. \\
& \left.-v_{k} \sum_{j=1}^{k} C_{2 k}^{2 j-1}(-1)^{k-j+1} t^{2 j-1}\right] \\
= & u_{k} \sum_{l=0}^{m-k} \sum_{j=0}^{k}(-1)^{k-j} C_{m-k}^{l} C_{2 k}^{2 j} t^{2(l+j)} \\
& +v_{k} \sum_{l=0}^{m-k} \sum_{j=1}^{k}(-1)^{k-j} C_{m-k}^{l} C_{2 k}^{2 j-1} t^{2(l+j)-1} .
\end{aligned}
$$

By making the index change $s:=l+j$, one gets

$$
\begin{aligned}
X_{k}(t)= & u_{k} \sum_{s=0}^{m} \sum_{\substack{0 \leq j \leq \min \{k, s\} \\
s-j \leq m-k}}(-1)^{k-j} C_{m-k}^{s-j} C_{2 k}^{2 j} t^{2 s} \\
& +v_{k} \sum_{s=1}^{m} \sum_{\substack{1 \leq j \leq \min \{k, s\} \\
s-j \leq m-k}}(-1)^{k-j} C_{m-k}^{s-j} C_{2 k}^{2 j-1} t^{2 s-1}
\end{aligned}
$$

so by (57) and (58) it follows that:

$$
X_{k}(t)=\sum_{s=0}^{m} u_{k} E_{s, k}^{m} t^{2 s}+\sum_{s=1}^{m} v_{k} F_{s, k}^{m} t^{2 s-1}
$$

for $1 \leq k \leq m$. Therefore, $E_{s, 0}^{m}=C_{m}^{s}$, we finally obtain

$$
\begin{aligned}
Q(t)= & \sum_{s=0}^{m} \frac{u_{0}}{2} C_{m}^{s} t^{2 s}+\sum_{s=0}^{m} \sum_{k=1}^{m} u_{k} E_{s, k}^{m} t^{2 s} \\
& +\sum_{s=1}^{m} \sum_{k=1}^{m} v_{k} F_{s, k}^{m} t^{2 s-1} \\
= & \sum_{s=0}^{m}\left[E_{s, 0}^{m} \frac{\delta_{0}}{2}+\sum_{k=0}^{m} u_{k} E_{s, k}^{m}\right] t^{2 s} \\
& +\sum_{s=1}^{m}\left[\sum_{k=1}^{m} v_{k} F_{s, k}^{n}\right] t^{2 s-1}
\end{aligned}
$$

and, therefore, by coefficient identification

$$
\begin{aligned}
q_{2 s} & =E_{s, 0}^{m} \frac{\delta_{0}}{2}+\sum_{k=1}^{m} E_{s, k}^{m} \operatorname{Re} \delta_{k} \quad(0 \leq s \leq m) \\
q_{2 s-1} & =\sum_{k=1}^{m} F_{s, k}^{m} \Im \delta_{k} \quad(1 \leq s \leq m)
\end{aligned}
$$

which shows (59).

\section{ACKNOWLEDGMENT}

The authors are indebted to the anonymous reviewers whose comments and suggestions improved the paper. They also would like to thank Prof. T. Kailath for his encouragement while carrying out this work.

\section{REFERENCES}

[1] O. Alata, M. Najim, C. Ramananjarasoa, and F. Turcu, "Extension of the Schur-Cohn stability test for 2-D AR quarter-plane model," IEEE Trans. Inf. Theory, vol. 49, no. 11, pp. 3099-3106, 2003.

[2] Y. Bistritz, "Zero location of polynomials with respect to the unit-circle unhampered by nonessential singularities," IEEE Trans. Circuits Syst. I. Reg. Papers, vol. 49, pp. 305-314, 2002.

[3] Y. Bistritz, "Testing stability of 2-D discrete systems by a set of real 1-D stability tests," IEEE Trans. Circuits Syst. I Reg. Papers, vol. 51, no. 7, pp. 1312-1320, 2004

[4] M. Bakonyi and T. Constantinescu, Schur's Algorithm and Several Applications, ser. Pitman Research Notes in Mathematics Series. Harlow: Longman Scientific and Technical, 1992, vol. 61

[5] N. K. Bose, Applied Multidimensional System Theory. New York: Van Nostrand Reinhold, 1982.

[6] N. K. Bose, Digital Filters: Theory and Applications. New York: North-Holland Elsevier, 1985.

[7] N. K. Bose, "Simplification of multidimensional digital filter stability test," J. Franklin Inst., vol. 330, no. 5, pp. 905-911, 1993.

[8] P. Fu, J. Chen, and S. I. Niculescu, "Generalized eigenvalue-based stability tests for 2-D linear systems: Necessary and sufficient conditions," Automatica, vol. 42, pp. 1569-1576, 2006.

[9] J. S. Geronimo and H. J. Woerdeman, "Two-variable polynomials: Intersecting zeros and stability," IEEE Trans. Circuits Syst. I, Reg. Papers, vol. 53, no. 5, pp. 1130-1139, 2006.

[10] E. I. Jury, Inners and Stability of Dynamic Systems. Malabar, FL: Robert Krieger, 1982.

[11] E. I. Jury, "Modified stability table for 2D digital filters," IEEE Trans. Circuits Syst., vol. 35, pp. 116-119, 1988.

[12] E. I. Jury, , S. Tzafestas, Ed., "Stability of multidimensional systems and other related problems," in Multidimensional Systems: Techniques and Applications. New York: Marcel Dekker, 1986, ch. 3, pp. 89-159.

[13] T. Kailath, "A theorem of I. Schur and its impact on modern signal processing," in I. Schur Methods in Operator Theory and Signal Processing. Boston, MA: Birkhauser Verlag, 1986, pp. 9-30.

[14] X. Liu and M. Najim, "A two-dimensional fast lattice recursive least squares algorithm," IEEE Trans. Signal Process., vol. 44, no. 10, pp. $2557-2567,1996$. 
[15] M. Marden, The Geometry of the Zeros of a Polynomial in a Complex Variable. New York: Amer. Math. Soc., 1949, mathematical surveys no. 3.

[16] S. Prajna, A. Papachristodoulou, P. Seiler, and P. Parrilo, Sums of Squares Optimization Toolbox for Matlab 2004 [Online]. Available: http://www.cds.caltech.edu/sostools, Version 2.00

[17] W. Rudin, Function Theory in Polydisks. New York-Amsterdam: W. A. Benjamin, 1969.

[18] I. Schur, "Über potenzreichen, die in innern des einheitskreises beschräNkt sind," J. Reine Angew. Math, vol. 147, 148, pp. 205-232, 1917, 122-145.

[19] I. Serban, F. Turcu, M. Najim, and Y. Stitou, "Multidimensional Schur coefficients and BIBO stability," Commun. Inf. Syst. (Special Issue to Honor of Prof. Thomas Kailath's 70th Birthday), vol. 5, no. 1, pp. 131-142, 2005.

[20] I. Serban, F. Turcu, and M. Najim, "Schur coefficients in several variables," J. Math. Anal. Appl., vol. 320, pp. 293-302, 2006.

[21] J. F. Sturm, SeDuMi, a Matlab Toolbox for Optimization over Symmetric Cones, Optimization Methods and Software vol. 11-12, pp. 625-653, 1999 [Online]. Available: http://fewcal.kub.nl/sturm/software/sedumi.html

[22] L. Xu, M. Yamada, Z. Lin, O. Saito, and Y. Anzawa, "Further improvements on Bose's 2D stability test," Int. J. Contr., Autom. Syst., vol. 2, no. 3, pp. 319-332, 2004.

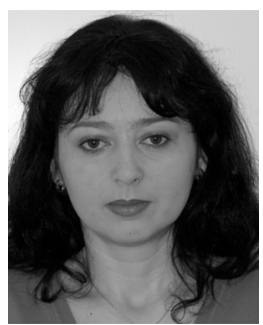

Ioana Serban was born on October 21, 1973, in Arad, Romania. She received the Ph.D. degree in pure mathematics (operator theory) from both the University Claude Bernard Lyon 1, France, and West University of Timisoara, Romania, in 2003.

Since 2004, she has been a Postdoctoral Researcher with the Department of Control and Signal Processing, IMS, Bordeaux 1, France. Her interests are in operator theory, several variables complex analysis, multidimensional system theory, and applications in multidimensional signal processing.

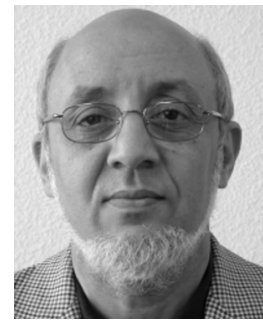

Mohamed Najim (M'74-SM'83-F'83) received the Dr. Sci. degree (Doctorat d'Etat) from the University of Toulouse, France, in 1972.

In 1972, he joined the University of Rabat, Morocco, as an Associate Professor, where he became a Professor in 1974. Since 1988, he has been a Professor with the ENSEIRB/University of Bordeaux, France, where he founded the Signal and Image Processing Laboratory. He has worked in various fields, including microwaves, modeling and identification, adaptive filtering including $\mathrm{H}$ infinity, and control. His research interests are presently modeling and identification in multidimensional signal and image processing with applications in speech, seismic, biomedical, radar signal processing, textures, and image enhancement. He supervised more than $50 \mathrm{Ph}$.D. theses and has published over 220 scientific papers and coauthored several books, including Parametric Modelling in Image Processing (Paris, France: Masson, 1994). He is author of Modelling and Identification in Signal Processing (Paris, France: Masson, 1988). He set up the "CAD Software Library" group within the GDR-CNRS TdSI, which is a French research program on signal and image processing.

Dr. Najim has organized 15 international conferences on control, signal processing, and friendly exchange through the Internet. He coorganized with Prof. T. Kailath (Stanford University) and Prof. P. Dewilde (Delft University) two workshops, respectively, dedicated to maths and systems (Saint Emilion, France, 1997) and structured algorithms (Cadzand, Netherlands, 2002). He was the Co-Chairman of the IEEE Statistical Signal Processing Conference ' 05 in Bordeaux, July 2005. He was the Editor of numerous symposia proceedings. From 1981 to 1990 , he was a member of the IFAC Technical Board. He is currently a member of the Technical Committee on Digital Signal Processing of the IEEE Circuits and Systems Society. Since 1999, he has been an elected Associate Member of the Third World Academy of Sciences. He has managed various projects with industrial partners (including Texas Instruments, Digital Equipment, ST Microelectronics). He is currently the head of the LASIS, a joint TOTAL/CNRS laboratory. 